\title{
THE ANGLO-AUSTRALIAN PLANET SEARCH. XXIII. TWO NEW JUPITER ANALOGS
}

\author{
Robert A. Wittenmyer ${ }^{1,2}$, Jonathan Horner ${ }^{1,2}$, C. G. Tinney ${ }^{1,2}$, R. P. Butler ${ }^{3}$, H. R. A. Jones ${ }^{4}$, Mikko Tuomi $^{4,5}$, \\ G. S. Salter ${ }^{1,2}$, B. D. CARTer ${ }^{6}$, F. Elliott Koch ${ }^{7}$, S. J. O’Toole ${ }^{8}$, J. Bailey ${ }^{1,2}$, And D. Wright ${ }^{1,2}$ \\ ${ }^{1}$ School of Physics, University of New South Wales, Sydney 2052, Australia; rob@ phys.unsw.edu.au \\ 2 Australian Centre for Astrobiology, University of New South Wales, Sydney 2052, Australia \\ ${ }^{3}$ Department of Terrestrial Magnetism, Carnegie Institution of Washington, 5241 Broad Branch Road, NW, Washington, DC 20015-1305, USA \\ ${ }^{4}$ University of Hertfordshire, Centre for Astrophysics Research, Science and Technology Research Institute, College Lane, AL10 9AB Hatfield, UK \\ ${ }^{5}$ University of Turku, Tuorla Observatory, Department of Physics and Astronomy, Väisäläntie 20, FI-21500 Piikkiö, Finland \\ ${ }^{6}$ Computational Engineering and Science Research Centre, University of Southern Queensland, Toowoomba, Queensland 4350, Australia \\ ${ }^{7}$ San Diego State University, Physics Department, 5500 Campanile Drive, San Diego, CA 92182-1233, USA \\ ${ }^{8}$ Australian Astronomical Observatory, P.O. Box 915, North Ryde, NSW 1670, Australia \\ Received 2013 December 22; accepted 2014 January 21; published 2014 February 20
}

\begin{abstract}
We report the discovery of two long-period giant planets from the Anglo-Australian Planet Search. HD 154857c is in a multiple-planet system, while HD 114613b appears to be solitary. HD $114613 \mathrm{~b}$ has an orbital period $P=10.5 \mathrm{yr}$, and a minimum mass $m \sin i$ of $0.48 M_{\text {Jup }}$ HD $154857 \mathrm{c}$ has $P=9.5 \mathrm{yr}$ and $m \sin i=2.6 M_{\text {Jup }}$. These new data confirm the planetary nature of the previously unconstrained long-period object in the HD 154857 system. We have performed detailed dynamical stability simulations which show that the HD 154857 two-planet system is stable on timescales of at least $10^{8} \mathrm{yr}$. These results highlight the continued importance of "legacy" surveys with long observational baselines; these ongoing campaigns are critical for determining the population of Jupiter analogs, and hence of those planetary systems with architectures most like our own solar system.
\end{abstract}

Key words: planets and satellites: detection - planets and satellites: dynamical evolution and stability planets and satellites: individual (HD 114613, HD 154857) - techniques: radial velocities

Online-only material: color figures

\section{INTRODUCTION}

A major theme that has unified exoplanet searches for more than $20 \mathrm{yr}$ is the question of how common (or rare) our own solar system is. The Kepler spacecraft, which continuously monitored over 100,000 stars for tiny eclipses caused by orbiting planets (Borucki et al. 2010), has provided exquisite data which have revolutionized our understanding of the frequency of Earth-size planets in short-period orbits (Howard et al. 2012; Fressin et al. 2013). However, Kepler alone cannot give us a complete picture of the occurrence rate of planetary systems like our own, with rocky inner planets and one or more gas giant planets ("Jupiter analogs") at orbital distances $a \gtrsim 3 \mathrm{AU}$. There is more to a system being solar system-like than having a single planet in a potentially habitable orbit. The detection of a Jupiter analog is a second key component in determining whether an exoplanetary system is solar-system-like. Over the years, many arguments have been put forth to suggest that such external giant planets might be a necessity for a potentially habitable exo-Earth to be a promising location for the development of life (Horner \& Jones 2010). Although the role of such planets acting as a shield from an otherwise damaging impact regime has come into question (e.g., Horner \& Jones 2008; Horner et al. 2010; Lewis et al. 2013), a number of other potential benefits are thought to accrue from the presence of Jupiteranalogs. For example, Jupiter-like planets have been proposed as a solution to the question of the origin of Earth's water. Current models of planetary formation suggest that the Earth formed in a region of the proto-planetary disk that was far too warm for water to condense from the gas phase. As such, it is challenging to explain the origin of our planet's water without invoking an exogenic cause. The formation and evolution of the giant planets, beyond the snow line, offers a natural explanation for the delivery of volatiles from the cold depths of a planetary system to planets that move on potentially habitable orbits (e.g., Horner et al. 2009; Horner \& Jones 2010 and references therein). The detection of a Jupiter-analog is therefore both a second key component in determining whether an exoplanetary system is solar-system-like, and a potential marker that the planets in that system might be promising targets for the future search for life beyond the solar system.

The Anglo-Australian Planet Search (AAPS) has been in operation for $15 \mathrm{yr}$, and has achieved a long-term radial-velocity precision of $3 \mathrm{~m} \mathrm{~s}^{-1}$ or better since its inception, which is enabling the detection of long-period giant planets. To date, the AAPS has discovered six Jupiter analogs: HD 70642b (Carter et al. 2003), HD 160691c (McCarthy et al. 2004), HD 30177b (Butler et al. 2006), GJ 832b (Bailey et al. 2009), HD 134987c (Jones et al. 2010), HD 142c (Wittenmyer et al. 2012c). Here, we have defined a Jupiter analog as a giant planet which has ended up near its formation location, beyond the ice line, with $a>3 \mathrm{AU}$. Recently, the AAPS has shifted its priority to the detection of these Jupiter analogs. The observing strategy and target list have been modified, with the aim of producing an accurate and precise determination of the frequency of Jupiterlike planets in Jupiter-like orbits (Wittenmyer et al. 2011b, 2013b). The modified target list includes stars with long-term velocity stability such that Jupiter analogs can be robustly excluded (e.g., Wittenmyer et al. 2006, 2011a), as well as those stars with as-yet-incomplete orbits suggestive of longperiod giant planets. In this paper, we report the discovery of two such Jupiter analogs with complete orbits. HD 154857 is already known to host a $1.8 M_{\text {Jup }}$ planet with an orbital period of about 400 days (McCarthy et al. 2004); a residual velocity trend indicated a much longer-period object, as noted in the discovery work and in O'Toole et al. (2007). 
This paper is organized as follows: Section 2 briefly describes the observational details and stellar parameters, and Section 3 details the orbit fitting process and gives the parameters of the two new planets. In Section 4, we present a dynamical stability analysis of the HD 154857 two-planet system, and we give our conclusions in Section 5.

\section{OBSERVATIONS AND STELLAR PARAMETERS}

AAPS Doppler measurements are made with the UCLES echelle spectrograph (Diego et al. 1990). An iodine absorption cell provides wavelength calibration from 5000 to $6200 \AA$. The spectrograph point-spread function (PSF) and wavelength calibration are derived from the iodine absorption lines embedded on the spectrum by the cell (Valenti et al. 1995; Butler et al. 1996). The result is a precise Doppler velocity estimate for each epoch, along with an internal uncertainty estimate, which includes the effects of photon-counting uncertainties, residual errors in the spectrograph PSF model, and variation in the underlying spectrum between the iodine-free template and epoch spectra observed through the iodine cell. All velocities are measured relative to the zero-point defined by the template observation. For HD 114613, a total of 223 Anglo-Australian Telescope (AAT) observations have been obtained since 1998 Jan 16 (Table 1) and used in the following analysis, representing a data span of 5636 days (15.4 yr). The mean internal velocity uncertainty for these data is $0.94 \mathrm{~m} \mathrm{~s}^{-1}$. HD 154857 has been observed 42 times since 2002 April (Table 2), for a total time span of 4109 days $(11.3 \mathrm{yr})$ and a mean internal uncertainty of $1.71 \mathrm{~m} \mathrm{~s}^{-1}$.

HD 114613 (HR 4979; HIP 64408) is an inactive G-type star, listed as a dwarf by Torres et al. (2006), though its surface gravity is more indicative of a slightly evolved subgiant (Table 3). It is a nearby and bright star $(V=4.85)$ with a somewhat super-solar metallicity $[\mathrm{Fe} / \mathrm{H}] \sim 0.19$. HD 154857 has been classified as a G5 dwarf (Houk \& Cowley 1975). However, all recent measurements of its surface gravity show that this star is a subgiant (Table 4). There is some confusion as to the mass: Valenti \& Fischer (2005) give two disparate mass estimates, $2.10 \pm 0.31 M_{\odot}$ derived from spectroscopic analysis, and $1.27_{-0.29}^{+0.35} M_{\odot}$ from interpolation on a grid of Yonsei-Yale isochrones. For most stars in their sample, the two mass estimates agreed within $\sim 10 \%$, but for HD 154857, they differ by almost a factor of two. The more recent analysis by Takeda et al. (2007) yields an intermediate value of $1.718_{-0.022}^{+0.03} M_{\odot}$, which we adopt in this paper.

\section{ORBIT FITTING AND PLANETARY PARAMETERS}

\subsection{HD 114613}

HD 114613 has been observed by the AAPS for the full $15 \mathrm{yr}$ of its operation. A long-period trend had been evident for several years, and in 2011, the trend resolved into a complete orbital cycle. We have since continued to observe HD 114613 to verify that the $\sim 11 \mathrm{yr}$ orbit was indeed turning around. Figure 2 shows the generalized Lomb-Scargle periodogram (Zechmeister \& Kürster 2009) of the 223 AAT observations. This type of periodogram weights the input data by their uncertainties, whereas the traditional Lomb-Scargle method (Lomb 1976; Scargle 1982) assumes uniform, Gaussian distributed uncertainties. To assess the significance of any signals appearing in these periodograms, we performed a bootstrap randomization process (Kürster et al. 1997). This randomly shuffles the velocity observations while keeping the times of observation fixed.
Table 1

AAT/UCLES Radial Velocities for HD 114613

\begin{tabular}{|c|c|c|}
\hline JD-2400000 & $\begin{array}{l}\text { Velocity } \\
\left(\mathrm{m} \mathrm{s}^{-1}\right)\end{array}$ & $\begin{array}{c}\text { Uncertainty } \\
\left(\mathrm{m} \mathrm{s}^{-1}\right)\end{array}$ \\
\hline 50830.25655 & 4.0 & 1.2 \\
\hline 50833.22381 & 1.9 & 0.8 \\
\hline 50915.08074 & 0.6 & 1.4 \\
\hline 50917.08662 & -7.5 & 1.6 \\
\hline 50970.91681 & -11.8 & 1.1 \\
\hline 51002.86733 & -5.6 & 1.8 \\
\hline 51212.26513 & 5.6 & 1.4 \\
\hline 51236.21281 & -5.5 & 1.4 \\
\hline 51237.18130 & -7.5 & 1.8 \\
\hline 51274.21875 & -1.8 & 2.0 \\
\hline 51276.08909 & -0.2 & 1.3 \\
\hline 51382.92476 & -3.9 & 1.2 \\
\hline 51386.85182 & 1.8 & 1.2 \\
\hline 51631.24051 & -1.9 & 1.3 \\
\hline 51682.84034 & 1.3 & 1.4 \\
\hline 51684.05975 & -2.8 & 1.4 \\
\hline 51717.83623 & -0.1 & 1.3 \\
\hline 51919.25519 & -0.8 & 1.8 \\
\hline 51920.26898 & -1.7 & 1.5 \\
\hline 51984.11127 & 0.3 & 1.7 \\
\hline 52061.03012 & 5.0 & 1.4 \\
\hline 52062.09081 & 3.8 & 1.5 \\
\hline 52092.95741 & 3.6 & 1.1 \\
\hline 52127.88681 & 0.4 & 1.7 \\
\hline 52359.20083 & 2.3 & 1.0 \\
\hline 52387.02810 & 4.4 & 1.4 \\
\hline 52388.06604 & 3.4 & 1.5 \\
\hline 52509.86723 & 13.4 & 1.3 \\
\hline 52510.86704 & 11.2 & 1.5 \\
\hline 52654.26882 & 4.6 & 1.4 \\
\hline 52710.14699 & 8.8 & 1.0 \\
\hline 52710.95867 & 6.4 & 2.0 \\
\hline 52745.09064 & 16.9 & 1.3 \\
\hline 52751.11506 & 9.0 & 1.4 \\
\hline 52752.07289 & 11.6 & 1.1 \\
\hline 52783.95975 & 11.8 & 1.2 \\
\hline 52785.05762 & 9.3 & 1.7 \\
\hline 52785.97105 & 15.2 & 1.4 \\
\hline 52857.87314 & 0.6 & 1.3 \\
\hline 53008.22116 & 11.7 & 1.3 \\
\hline 53041.22754 & 8.0 & 1.3 \\
\hline 53042.22343 & 4.2 & 1.2 \\
\hline 53046.15053 & 5.2 & 1.5 \\
\hline 53051.15875 & -0.3 & 1.0 \\
\hline 53214.87828 & 10.8 & 1.0 \\
\hline 53215.88215 & 9.6 & 1.2 \\
\hline 53242.89915 & 4.5 & 0.8 \\
\hline 53245.84896 & 14.4 & 1.5 \\
\hline 53399.27632 & 5.5 & 0.6 \\
\hline 53405.20308 & 2.0 & 0.7 \\
\hline 53482.95081 & -1.3 & 0.8 \\
\hline 53484.04090 & -4.9 & 0.7 \\
\hline 53485.02218 & -0.9 & 0.7 \\
\hline 53485.94007 & 0.0 & 0.7 \\
\hline 53486.06578 & -0.8 & 0.7 \\
\hline 53488.12718 & 1.2 & 0.7 \\
\hline 53489.07405 & -0.4 & 0.7 \\
\hline 53506.95930 & 1.5 & 0.7 \\
\hline 53507.88240 & 0.4 & 0.7 \\
\hline 53509.07069 & -4.2 & 0.7 \\
\hline 53516.02470 & 2.0 & 1.0 \\
\hline 53517.00282 & 6.4 & 0.8 \\
\hline 53518.95553 & 6.2 & 0.7 \\
\hline 53520.02670 & 6.0 & 0.8 \\
\hline 53521.01119 & 7.7 & 0.8 \\
\hline
\end{tabular}


Table 1

(Continued)

\begin{tabular}{|c|c|c|}
\hline JD-2400000 & $\begin{array}{l}\text { Velocity } \\
\left(\mathrm{m} \mathrm{s}^{-1}\right)\end{array}$ & $\begin{array}{l}\text { Uncertainty } \\
\left(\mathrm{m} \mathrm{s}^{-1}\right)\end{array}$ \\
\hline 53521.93055 & 4.4 & 0.9 \\
\hline 53522.97922 & 4.0 & 0.8 \\
\hline 53568.93024 & 1.4 & 0.7 \\
\hline 53569.89040 & -2.5 & 0.8 \\
\hline 53570.93229 & -0.1 & 0.7 \\
\hline 53571.92818 & 4.4 & 0.7 \\
\hline 53572.93960 & 3.5 & 0.6 \\
\hline 53573.86024 & 2.8 & 0.7 \\
\hline 53575.86501 & 4.8 & 0.6 \\
\hline 53576.83855 & 1.5 & 0.6 \\
\hline 53577.85712 & 0.4 & 0.9 \\
\hline 53578.87161 & 1.6 & 0.6 \\
\hline 53840.18077 & -2.4 & 0.9 \\
\hline 53841.14463 & 2.1 & 0.8 \\
\hline 53843.11871 & 0.4 & 0.8 \\
\hline 53844.06189 & 1.7 & 0.7 \\
\hline 53937.92281 & -1.6 & 0.7 \\
\hline 53938.90015 & 1.4 & 0.6 \\
\hline 53943.85615 & -4.9 & 0.6 \\
\hline 53944.87540 & -7.3 & 0.6 \\
\hline 53945.86861 & -4.3 & 0.8 \\
\hline 53946.86415 & -7.3 & 0.6 \\
\hline 54111.19771 & 3.5 & 0.6 \\
\hline 54112.20754 & 4.7 & 0.8 \\
\hline 54113.22282 & 3.5 & 0.8 \\
\hline 54114.24651 & 2.6 & 0.9 \\
\hline 54115.25260 & 3.5 & 1.2 \\
\hline 54120.20490 & 7.7 & 0.7 \\
\hline 54121.19705 & 3.8 & 0.6 \\
\hline 54123.22186 & 9.1 & 0.6 \\
\hline 54126.18385 & 2.7 & 0.7 \\
\hline 54127.18893 & 5.0 & 0.5 \\
\hline 54128.18767 & 4.0 & 0.8 \\
\hline 54129.18497 & -0.7 & 0.5 \\
\hline 54130.17738 & 0.9 & 0.7 \\
\hline 54131.18561 & 2.0 & 0.6 \\
\hline 54132.19227 & 2.1 & 0.8 \\
\hline 54133.25166 & 1.4 & 1.1 \\
\hline 54134.21614 & 4.1 & 0.6 \\
\hline 54135.18151 & -1.1 & 0.7 \\
\hline 54136.20085 & 0.8 & 0.6 \\
\hline 54137.19842 & 1.3 & 0.6 \\
\hline 54138.17883 & 0.1 & 0.9 \\
\hline 54139.17104 & 3.0 & 0.8 \\
\hline 54140.17481 & -0.1 & 0.8 \\
\hline 54141.20213 & -1.6 & 0.8 \\
\hline 54142.19235 & 0.6 & 0.5 \\
\hline 54144.12894 & -3.4 & 0.6 \\
\hline 54145.15798 & -2.9 & 0.6 \\
\hline 54146.17863 & -0.3 & 0.6 \\
\hline 54147.19558 & -3.5 & 0.6 \\
\hline 54148.22623 & -2.7 & 0.6 \\
\hline 54149.16439 & -2.0 & 0.6 \\
\hline 54150.19348 & -1.7 & 0.6 \\
\hline 54151.20779 & -3.0 & 0.6 \\
\hline 54152.22033 & -2.7 & 0.7 \\
\hline 54154.19334 & -5.2 & 0.6 \\
\hline 54155.19519 & -3.2 & 0.7 \\
\hline 54156.16473 & -1.3 & 0.6 \\
\hline 54223.10365 & 1.9 & 0.9 \\
\hline 54224.14365 & 1.1 & 0.8 \\
\hline 54225.07630 & 1.8 & 0.7 \\
\hline 54226.00805 & 1.9 & 1.0 \\
\hline 54227.04258 & 5.5 & 0.8 \\
\hline 54252.96645 & 9.5 & 0.9 \\
\hline
\end{tabular}

Table 1

(Continued)

\begin{tabular}{|c|c|c|}
\hline JD-2400000 & $\begin{array}{l}\text { Velocity } \\
\left(\mathrm{m} \mathrm{s}^{-1}\right)\end{array}$ & $\begin{array}{l}\text { Uncertainty } \\
\left(\mathrm{m} \mathrm{s}^{-1}\right)\end{array}$ \\
\hline 54254.00991 & 9.0 & 0.8 \\
\hline 54254.91285 & 5.6 & 0.9 \\
\hline 54255.97533 & 3.7 & 0.9 \\
\hline 54257.06303 & 5.6 & 0.9 \\
\hline 54333.85357 & 3.5 & 0.9 \\
\hline 54334.87415 & 2.6 & 0.9 \\
\hline 54335.86542 & 2.5 & 0.7 \\
\hline 54336.85846 & 6.7 & 0.8 \\
\hline 54543.05240 & 0.0 & 0.8 \\
\hline 54544.14396 & -0.8 & 0.8 \\
\hline 54550.09556 & -1.0 & 1.4 \\
\hline 54551.09203 & -1.9 & 0.9 \\
\hline 54552.13245 & 3.0 & 1.1 \\
\hline 54553.10244 & -3.6 & 1.1 \\
\hline 54841.25120 & -0.4 & 1.0 \\
\hline 54897.20442 & -8.4 & 1.0 \\
\hline 54900.18137 & -12.3 & 1.2 \\
\hline 54901.15823 & -6.8 & 0.9 \\
\hline 54902.20552 & -10.1 & 1.0 \\
\hline 54904.19780 & -6.0 & 1.0 \\
\hline 54905.26523 & -1.6 & 0.8 \\
\hline 54906.21006 & -5.8 & 1.0 \\
\hline 54907.19989 & -6.8 & 0.9 \\
\hline 54908.20498 & -6.8 & 0.8 \\
\hline 55014.94187 & -6.2 & 1.0 \\
\hline 55015.86595 & -4.9 & 0.8 \\
\hline 55018.84451 & 1.4 & 1.9 \\
\hline 55019.87410 & -5.8 & 0.7 \\
\hline 55020.84762 & -6.3 & 0.7 \\
\hline 55021.87399 & -7.7 & 0.8 \\
\hline 55022.88779 & -10.4 & 0.8 \\
\hline 55023.86739 & -11.0 & 0.9 \\
\hline 55029.84869 & -6.6 & 0.6 \\
\hline 55030.83778 & -4.0 & 0.7 \\
\hline 55031.90051 & -3.4 & 0.7 \\
\hline 55032.91264 & -3.6 & 0.6 \\
\hline 55036.84785 & -6.2 & 0.7 \\
\hline 55037.83638 & -2.9 & 0.7 \\
\hline 55040.84113 & -6.1 & 0.8 \\
\hline 55041.85626 & -6.0 & 0.9 \\
\hline 55043.88548 & -3.7 & 0.6 \\
\hline 55044.85993 & -4.9 & 0.6 \\
\hline 55045.85496 & -5.0 & 0.5 \\
\hline 55046.90149 & -6.5 & 0.7 \\
\hline 55047.88023 & -4.0 & 0.5 \\
\hline 55048.86964 & -5.4 & 0.5 \\
\hline 55049.85230 & -4.1 & 1.0 \\
\hline 55050.85220 & -7.8 & 0.8 \\
\hline 55051.84966 & -5.0 & 0.5 \\
\hline 55053.85289 & -8.7 & 0.6 \\
\hline 55054.84736 & -8.0 & 0.6 \\
\hline 55055.88706 & -6.2 & 0.5 \\
\hline 55058.87417 & -4.9 & 0.6 \\
\hline 55206.19055 & -2.4 & 0.8 \\
\hline 55209.20056 & -1.9 & 0.8 \\
\hline 55253.19939 & -6.9 & 0.9 \\
\hline 55254.28349 & -9.6 & 1.0 \\
\hline 55310.06142 & 2.1 & 0.8 \\
\hline 55312.07035 & -2.4 & 0.8 \\
\hline 55317.03644 & -5.0 & 0.9 \\
\hline 55370.94153 & 3.1 & 1.0 \\
\hline 55374.90773 & 0.7 & 1.0 \\
\hline 55397.89284 & -6.3 & 1.0 \\
\hline 55402.84315 & -5.0 & 0.7 \\
\hline 55586.21266 & 0.8 & 0.9 \\
\hline
\end{tabular}


Table 1

(Continued)

\begin{tabular}{|c|c|c|}
\hline JD-2400000 & $\begin{array}{l}\text { Velocity } \\
\left(\mathrm{m} \mathrm{s}^{-1}\right)\end{array}$ & $\begin{array}{l}\text { Uncertainty } \\
\left(\mathrm{m} \mathrm{s}^{-1}\right)\end{array}$ \\
\hline 55603.27577 & 3.8 & 1.1 \\
\hline 55604.27940 & -0.2 & 1.1 \\
\hline 55663.95088 & 0.5 & 1.1 \\
\hline 55666.06790 & 2.5 & 0.9 \\
\hline 55692.08906 & 0.5 & 1.1 \\
\hline 55692.97915 & 5.8 & 0.9 \\
\hline 55750.89289 & -3.6 & 0.8 \\
\hline 55751.87344 & -2.2 & 1.3 \\
\hline 55753.84964 & -3.0 & 1.0 \\
\hline 55756.87098 & -1.2 & 1.1 \\
\hline 55785.90110 & 2.3 & 1.0 \\
\hline 55786.86812 & 4.9 & 1.5 \\
\hline 55787.92128 & 5.1 & 1.1 \\
\hline 55961.17818 & -0.1 & 0.8 \\
\hline 55964.22153 & 6.2 & 0.8 \\
\hline 55996.09776 & -3.3 & 0.9 \\
\hline 56049.03768 & 2.1 & 1.1 \\
\hline 56051.03503 & -0.1 & 1.1 \\
\hline 56084.99567 & 9.3 & 1.3 \\
\hline 56085.98717 & 3.6 & 1.1 \\
\hline 56134.98050 & 6.9 & 1.2 \\
\hline 56138.89373 & 3.4 & 1.1 \\
\hline 56379.13086 & 2.6 & 1.3 \\
\hline 56382.21632 & 10.6 & 1.9 \\
\hline 56383.07275 & 1.5 & 0.8 \\
\hline 56464.91329 & 10.2 & 1.1 \\
\hline 56465.92701 & 9.3 & 0.9 \\
\hline 56466.91347 & 8.0 & 0.9 \\
\hline
\end{tabular}

The periodogram of this shuffled data set is then computed and its highest peak recorded. The longest-period peak near 4000 days is well-defined and highly significant, with a bootstrap false-alarm probability less than $10^{-5}$. The next-highest peaks are at 122 and 1400 days, respectively. We fit these data with a single, long-period Keplerian using the GaussFit (Jefferys et al. 1988) nonlinear least-squares minimization routine. Jitter of $3.42 \mathrm{~m} \mathrm{~s}^{-1}$ (Wright 2005; O'Toole et al. 2009a) was added in quadrature to the uncertainties at each epoch prior to orbit fitting. A single-planet fit yields a period $P=3825 \pm$ 106 days, $K=5.4 \pm 0.4 \mathrm{~m} \mathrm{~s}^{-1}$, and $e=0.25 \pm 0.08$ (Table 5), making this planet a Jupiter analog (Wittenmyer et al. $2011 b$ ), with a minimum mass $m \sin i$ of $0.5 M_{\text {Jup }}$, and an orbital period of $10.7 \mathrm{yr}$ (Figure 1). The rms about the one-planet fit is $3.9 \mathrm{~m} \mathrm{~s}^{-1}$, and the periodogram of the residuals to this fit is shown in the right panel of Figure 2, showing a number of peaks ranging from 28 to $\sim 1500$ days.

There is structure evident in this residual periodogram (Figure 2, right panel), so we examined the residuals for additional Keplerian signals. One way of determining the veracity of such signals is to examine the data by seasons or subsets. This can disentangle true planetary signals (which would consistently appear in all subsets) from stochastic signals such as stellar rotational modulation (Dumusque et al. 2012; Hatzes 2013). We divided the residuals to the one-planet fit into two eight-season chunks. HD 114613 was observed intensely in 2007 and 2009 as part of the AAT "Rocky Planet Search" campaigns (O'Toole et al. 2009a, 2009b), in which 24-30 bright stars were observed nightly for 48 continuous nights in search of short-period planets. It is possible that such a density of observational data may skew the false-alarm probabilities when evaluating potential
Table 2

AAT/UCLES Radial Velocities for HD 154857

\begin{tabular}{|c|c|c|}
\hline JD-2400000 & $\begin{array}{l}\text { Velocity } \\
\left(\mathrm{m} \mathrm{s}^{-1}\right)\end{array}$ & $\begin{array}{c}\text { Uncertainty } \\
\left(\mathrm{m} \mathrm{s}^{-1}\right)\end{array}$ \\
\hline 52389.23580 & -3.3 & 1.7 \\
\hline 52390.21223 & -4.9 & 1.5 \\
\hline 52422.13713 & -17.3 & 1.5 \\
\hline 52453.01957 & -14.2 & 1.4 \\
\hline 52455.02535 & -13.7 & 1.9 \\
\hline 52455.97664 & -12.2 & 1.9 \\
\hline 52509.94853 & -16.3 & 2.1 \\
\hline 52510.91619 & -6.6 & 1.8 \\
\hline 52711.24602 & 63.7 & 2.8 \\
\hline 52745.24271 & 66.4 & 1.9 \\
\hline 52747.21175 & 58.7 & 1.8 \\
\hline 52750.17761 & 52.7 & 1.5 \\
\hline 52751.22944 & 47.2 & 1.4 \\
\hline 52784.12626 & -11.5 & 1.3 \\
\hline 52857.02974 & -28.4 & 2.7 \\
\hline 52857.98599 & -31.9 & 1.4 \\
\hline 52942.91225 & -18.1 & 1.7 \\
\hline 53217.01252 & -41.2 & 1.5 \\
\hline 53246.03809 & -53.3 & 1.9 \\
\hline 53485.15229 & 21.5 & 1.5 \\
\hline 53510.15968 & 25.9 & 1.4 \\
\hline 53523.10133 & 33.2 & 1.5 \\
\hline 53570.02945 & 32.9 & 2.3 \\
\hline 53843.23961 & -7.0 & 1.6 \\
\hline 53945.03237 & 49.6 & 1.0 \\
\hline 54008.89626 & -21.4 & 0.9 \\
\hline 54037.88134 & -39.5 & 1.4 \\
\hline 54156.28808 & -17.5 & 1.9 \\
\hline 54226.21678 & -2.3 & 1.2 \\
\hline 54254.99229 & 5.5 & 1.6 \\
\hline 54372.93185 & 61.1 & 1.3 \\
\hline 54552.23398 & -9.0 & 2.2 \\
\hline 54901.28209 & -7.9 & 2.1 \\
\hline 55101.89348 & 54.8 & 1.7 \\
\hline 55313.23386 & 2.5 & 1.4 \\
\hline 55317.13834 & 1.1 & 1.7 \\
\hline 55399.04324 & 17.8 & 1.4 \\
\hline 55429.91942 & 23.9 & 1.3 \\
\hline 56049.24780 & 1.1 & 2.1 \\
\hline 56465.07924 & -22.4 & 1.9 \\
\hline 56467.06137 & -20.2 & 1.7 \\
\hline 56498.02816 & -40.6 & 3.0 \\
\hline
\end{tabular}

additional signals. We thus removed the 66 epochs from the two "Rocky Planet Search" campaigns - this resulted in the 8 yr halves containing 77 and 80 observations, respectively. ${ }^{9}$ Periodograms of the two halves are shown in Figure 3; visual inspection reveals that they are markedly different.

Table 6 shows the false-alarm probabilities obtained from 10,000 such realizations on each half of the one-planet residuals. No periodicity is consistently significant in both subsets, with the possible exception of that near 27-29 days-however, this is worryingly close to both the 33 day rotation period of the star (Saar \& Osten 1997) and the lunar month (at which the sampling of radial-velocity observations is well-known to impart spurious periodicities, e.g., Dawson \& Fabrycky 2010; Wittenmyer et al. $2013 b$ ). While it is tempting to consider a second planet near 1400 days, as found in the raw-data periodogram (Figure 2),

\footnotetext{
9 Fitting a single planet with this shortened data set gives parameters within $1 \sigma$ of those given in Table 5, showing that the exclusion of those data do not affect our conclusions about the long-period planet.
} 
Table 3

Stellar Parameters for HD 114613

\begin{tabular}{lcl}
\hline \hline Parameter & Value & \multicolumn{1}{c}{ Reference } \\
\hline Spec. type & G4IV & Gray et al. (2006) \\
& G3V & Torres et al. (2006) \\
Mass $\left(M_{\odot}\right)$ & 1.364 & Sousa et al. (2008) \\
Distance $(\mathrm{pc})$ & $20.67 \pm 0.13$ & van Leeuwen (2007) \\
$V \sin i\left(\mathrm{~km} \mathrm{~s}^{-1}\right)$ & $2.7 \pm 0.9$ & Saar \& Osten (1997) \\
$\log R_{H K}^{\prime}$ & -5.118 & Gray et al. (2006) \\
{$[\mathrm{Fe} / \mathrm{H}]$} & $0.19 \pm 0.01$ & Sousa et al. (2008) \\
& 0.18 & Maldonado et al. (2012) \\
{$[\mathrm{O} / \mathrm{H}]$} & $0.03 \pm 0.01$ & Bond et al. (2008) \\
{$[\mathrm{Cr} / \mathrm{H}]$} & $0.09 \pm 0.04$ & Bond et al. (2008) \\
{$[\mathrm{Mg} / \mathrm{H}]$} & $-0.04 \pm 0.06$ & Bond et al. (2008) \\
{$[\mathrm{Zr} / \mathrm{H}]$} & $0.17 \pm 0.04$ & Bond et al. (2008) \\
{$[\mathrm{Eu} / \mathrm{H}]$} & $-0.17 \pm 0.04$ & Bond et al. (2008) \\
{$[\mathrm{Nd} / \mathrm{H}]$} & $0.05 \pm 0.01$ & Bond et al. (2008) \\
{$[\mathrm{Si} / \mathrm{H}]$} & $0.19 \pm 0.06$ & Bond et al. (2006) \\
$T_{\text {eff }}(\mathrm{K})$ & $5729 \pm 17$ & Sousa et al. (2008) \\
& 5574 & Gray et al. (2006) \\
$\log g$ & 5550 & Saar \& Osten (1997) \\
& $3.97 \pm 0.02$ & Sousa et al. (2008) \\
& 3.90 & Gray et al. (2006) \\
\hline
\end{tabular}

Table 4

Stellar Parameters for HD 154857

\begin{tabular}{|c|c|c|}
\hline Parameter & Value & Reference \\
\hline Spec. type & G5V & Houk \& Cowley (1975) \\
\hline Mass $\left(M_{\odot}\right)$ & $1.718_{-0.022}^{+0.03}$ & Takeda et al. (2007) \\
\hline Distance (pc) & $64.2 \pm 3.1$ & van Leeuwen (2007) \\
\hline$V \sin i\left(\mathrm{~km} \mathrm{~s}^{-1}\right)$ & $1.4 \pm 0.5$ & Butler et al. (2006) \\
\hline \multirow[t]{2}{*}{$\log R_{H K}^{\prime}$} & -5.00 & Jenkins et al. (2006) \\
\hline & -5.14 & Henry et al. (1996) \\
\hline \multirow[t]{4}{*}[\mathrm{Fe}/\mathrm{H}]{} & -0.31 & Holmberg et al. (2009) \\
\hline & -0.30 & Ghezzi et al. (2010) \\
\hline & -0.22 & Valenti \& Fischer (2005) \\
\hline & -0.20 & Casagrande et al. (2011) \\
\hline$[\mathrm{O} / \mathrm{H}]$ & $-0.15 \pm 0.03$ & Bond et al. (2008) \\
\hline$[\mathrm{Cr} / \mathrm{H}]$ & $-0.20 \pm 0.04$ & Bond et al. (2008) \\
\hline$[\mathrm{Mg} / \mathrm{H}]$ & $-0.20 \pm 0.03$ & Bond et al. (2008) \\
\hline$[\mathrm{Zr} / \mathrm{H}]$ & $-0.08 \pm 0.04$ & Bond et al. (2008) \\
\hline$[\mathrm{Eu} / \mathrm{H}]$ & $-0.27 \pm 0.07$ & Bond et al. (2008) \\
\hline$[\mathrm{Nd} / \mathrm{H}]$ & $-0.01 \pm 0.02$ & Bond et al. (2008) \\
\hline$[\mathrm{C} / \mathrm{H}]$ & $-0.28 \pm 0.07$ & Bond et al. (2006) \\
\hline$[\mathrm{Si} / \mathrm{H}]$ & $-0.28 \pm 0.11$ & Bond et al. (2006) \\
\hline \multirow[t]{3}{*}{$T_{\text {eff }}(\mathrm{K})$} & 5605 & Dodson-Robinson et al. (2011) \\
\hline & 5508 & Holmberg et al. (2009) \\
\hline & 5548 & Ghezzi et al. (2010) \\
\hline \multirow[t]{3}{*}{$\log g$} & $3.95_{-0.03}^{+0.05}$ & Takeda et al. (2007) \\
\hline & 3.82 & Ghezzi et al. (2010) \\
\hline & $3.99 \pm 0.06$ & Valenti \& Fischer (2005) \\
\hline \multirow[t]{2}{*}{ Radius $\left(R_{\odot}\right)$} & $2.31_{-0.10}^{+0.17}$ & Takeda et al. (2007) \\
\hline & $1.760 \pm 0.057$ & Torres et al. (2010) \\
\hline
\end{tabular}

we see that this signal is simply not evident in the first $8 \mathrm{yr}$ of observations. As suggested by Hatzes (2013) for the proposed planet orbiting Alpha Centauri B, we rephrase his sentiments to

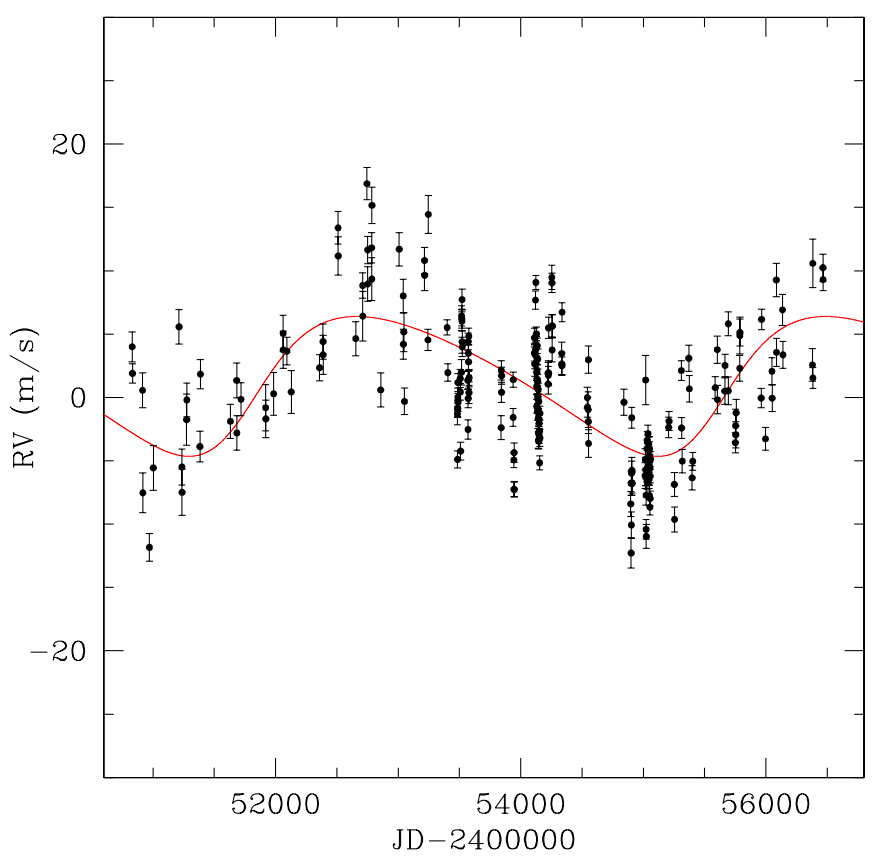

Figure 1. Keplerian orbit fit for HD 114613b. The planet has completed about 1.5 cycles, and the AAT data show a residual rms scatter of $3.9 \mathrm{~m} \mathrm{~s}^{-1}$.

(A color version of this figure is available in the online journal.)

Table 6

Candidate Secondary Signals for HD 114613

\begin{tabular}{lccc}
\hline \hline $\begin{array}{l}\text { First Half } \\
\text { Period (days) }\end{array}$ & FAP & $\begin{array}{c}\text { Second Half } \\
\text { Period (days) }\end{array}$ & FAP \\
\hline 28.9 & 0.015 & 26.6 & 0.0001 \\
72.5 & 0.952 & 73.3 & 0.1836 \\
122 & 1.000 & 121.4 & 0.0246 \\
490.2 & 0.138 & 480.8 & 0.9755 \\
1562.5 & 0.910 & 1111.1 & 0.0003
\end{tabular}

express that any shorter period periodic signal which is evident in one subset of our data should also be evident in other subsets or seasons of the data. As both HD 114613 eight-year subsets have ample time coverage and data quantity $(N=77)$ to sample the candidate periods listed in Table 6 , we can use these results to conclude that there is not yet sufficient evidence for additional planetary signals in our data for HD 114613.

\section{2. $H D 154857$}

The presence of a planet orbiting HD 154857 was first reported by McCarthy et al. (2004), who noted that the AAPS data were best fit with the $\sim 400$ day planet and a linear trend, indicating a more distant body. Additional data presented in O'Toole et al. (2007) refined the planet's parameters and attempted to constrain the outer object's orbit since the residual velocity trend had begun to show curvature. They determined a minimum orbit with period 1900 days and $K \sim 23 \mathrm{~m} \mathrm{~s}^{-1}$.

Table 5

Keplerian Orbital Solutions

\begin{tabular}{|c|c|c|c|c|c|c|c|c|}
\hline Planet & $\begin{array}{l}\text { Period } \\
\text { (days) }\end{array}$ & $\begin{array}{c}T_{0} \\
\text { (JD-2400000) }\end{array}$ & $e$ & $\begin{array}{c}\omega \\
(\operatorname{deg})\end{array}$ & $\begin{array}{c}K \\
\left(\mathrm{~m} \mathrm{~s}^{-1}\right)\end{array}$ & $\begin{array}{l}m \sin i \\
\left(M_{\text {Jup }}\right)\end{array}$ & $\begin{array}{c}a \\
(\mathrm{AU})\end{array}$ & $\chi_{v}^{2}$ \\
\hline HD 114613 b & $3827 \pm 105$ & $55550.3 \pm$ (fixed) & $0.25 \pm 0.08$ & $244 \pm 5$ & $5.52 \pm 0.40$ & $0.48 \pm 0.04$ & $5.16 \pm 0.13$ & 1.23 \\
\hline HD $154857 \mathrm{~b}$ & $408.6 \pm 0.5$ & $53572.5 \pm 2.4$ & $0.46 \pm 0.02$ & $57 \pm 4$ & $48.3 \pm 1.0$ & $2.24 \pm 0.05$ & $1.291 \pm 0.008$ & 1.35 \\
\hline HD $154857 \mathrm{c}$ & $3452 \pm 105$ & $55219 \pm 375$ & $0.06 \pm 0.05$ & $352 \pm 37$ & $24.2 \pm 1.1$ & $2.58 \pm 0.16$ & $5.36 \pm 0.09$ & \\
\hline
\end{tabular}



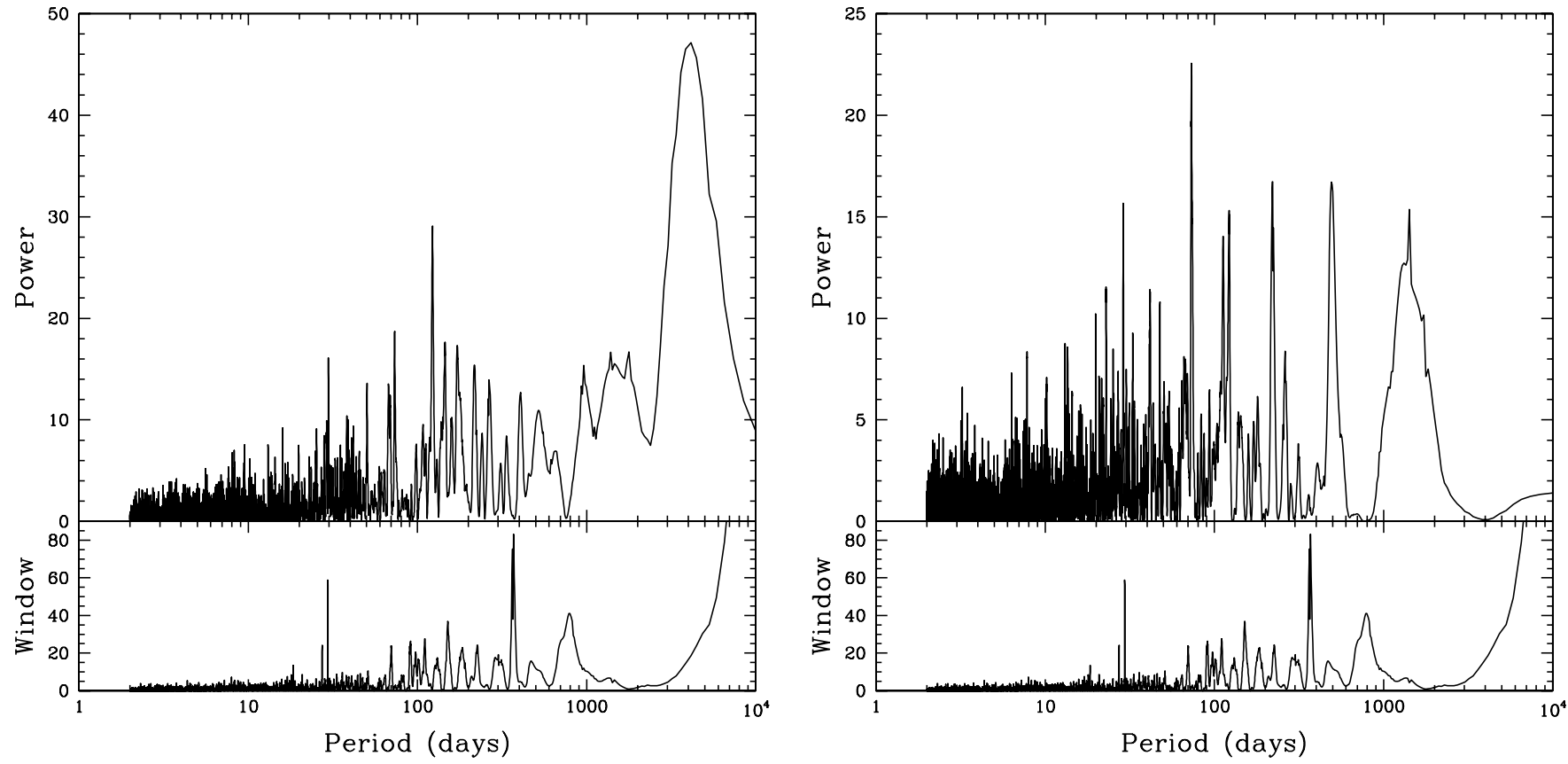

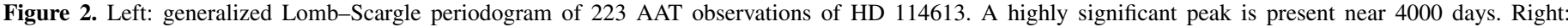

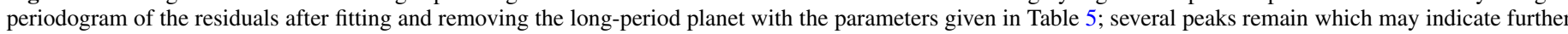
planets.
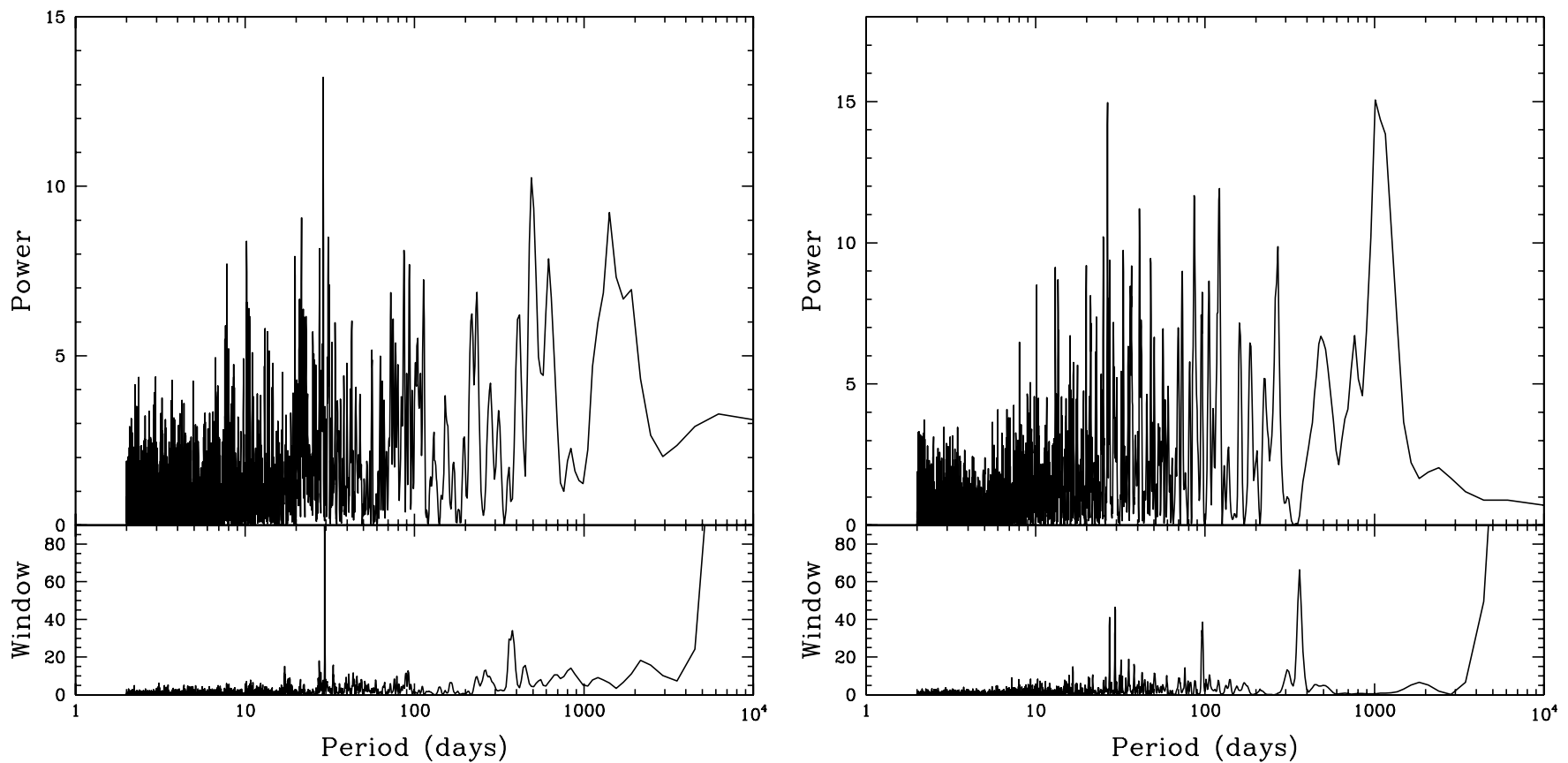

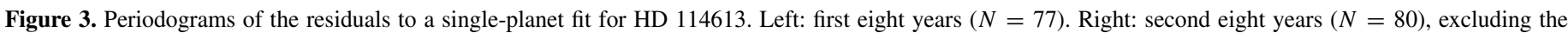
high-cadence observing runs in 2007 and 2009.

Now, with a further $6 \mathrm{yr}$ of AAT data, the outer planet has completed an orbit and a double-Keplerian model converges easily. We used GaussFit as described above to fit the two planets, first adding jitter of $2.6 \mathrm{~m} \mathrm{~s}^{-1}$ in quadrature to the uncertainties (after O'Toole et al. 2007). The best-fit parameters are given in Table 5; the outer planet is a Jupiter analog moving on an essentially circular orbit with $P=9.5 \mathrm{yr}(a=5.36 \mathrm{AU})$ and $m \sin i=2.6 M_{\text {Jup }}$. The rms about the two-planet fit is $3.2 \mathrm{~m} \mathrm{~s}^{-1}$, and there are no significant residual periodicities. The data and two-planet model are shown in Figure 4, and the orbital fits for the individual planets are shown in Figure 5.

\section{DYNAMICAL STABILITY TESTING}

Recent work has shown that any claim of multiple orbiting bodies must be checked by dynamical stability testing to ensure that the proposed planetary orbits are feasible on astronomically relevant timescales. Such testing can support the orbit fitting results (Robertson et al. 2012a; Wittenmyer et al. 2012c; Horner et al. 2012b), place further constraints on the planetary system configurations (Robertson et al. 2012b; Wittenmyer et al. 2012b; Tan et al. 2013), or show that the proposed planets cannot exist in or near the nominal best-fit orbits (Wittenmyer et al. 


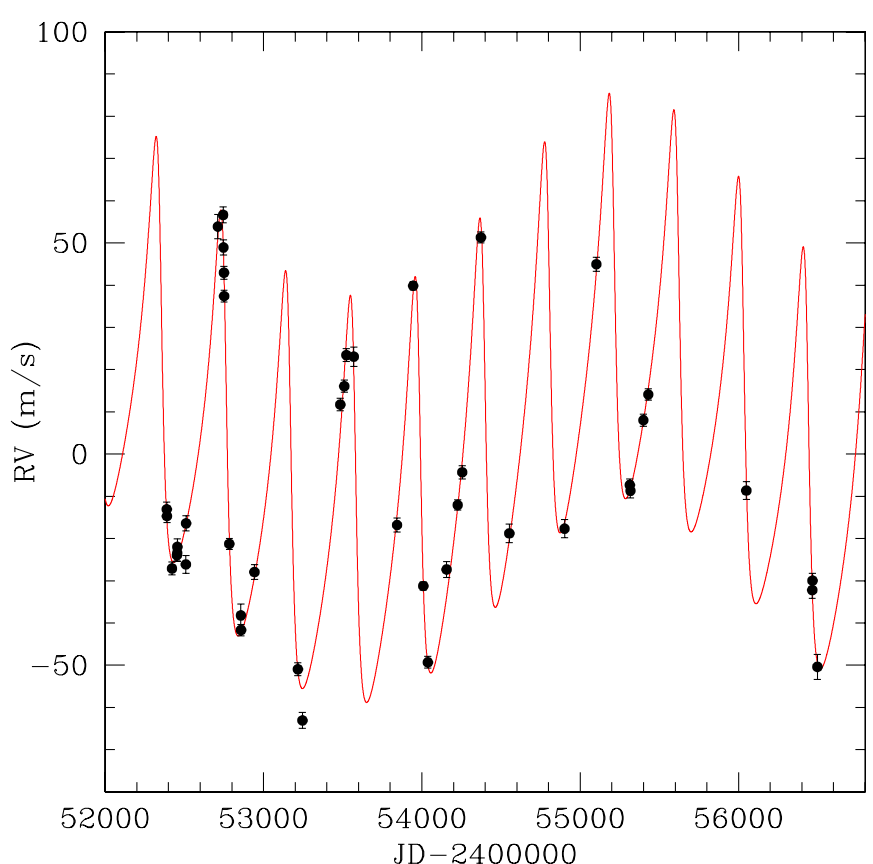

Figure 4. Two-planet Keplerian model and AAT data for the HD 154857 system. The rms about this fit is $3.2 \mathrm{~m} \mathrm{~s}^{-1}$.

(A color version of this figure is available in the online journal.)

2012a, 2013a; Goździewski et al. 2012; Horner et al. 2012a, 2013). While HD 114613 appears to be a single-planet system, HD 154857 hosts two planets which are so widely separated (1.3 and $5.4 \mathrm{AU}$ ) that their dynamical interactions might be expected to be negligible. However, for completeness, we performed the dynamical analysis as in our previous work (Wittenmyer et al. 2012c).

We tested the dynamical stability of the HD 154857 system using the Hybrid integrator within the $n$-body dynamics package Mercury (Chambers 1999). Holding the initial orbit of the

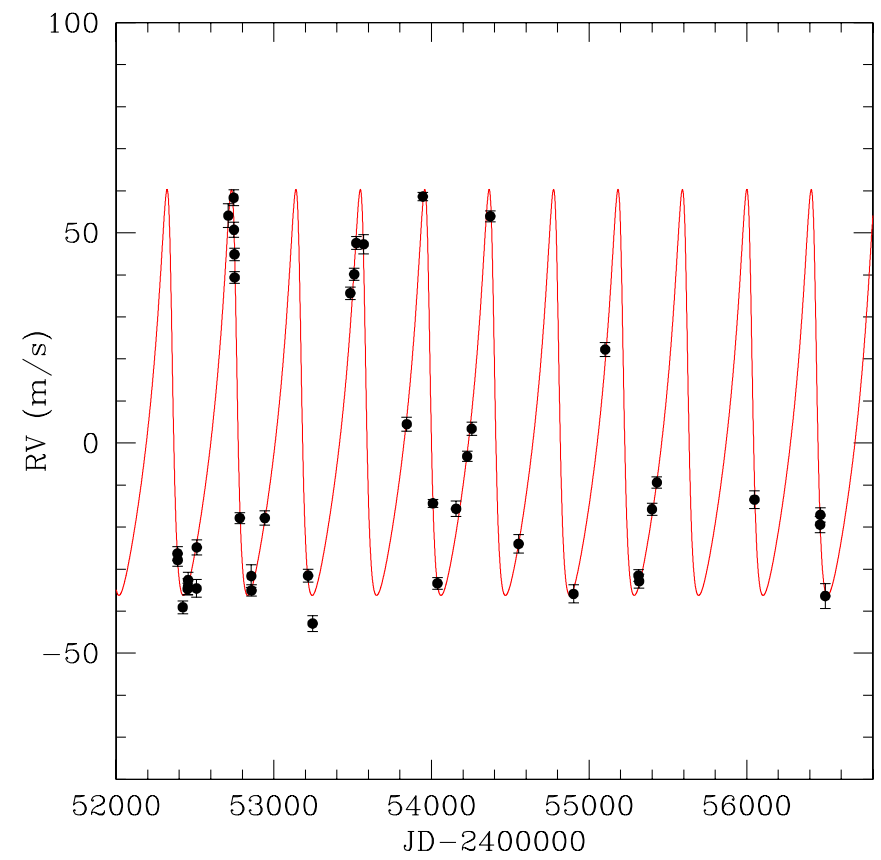

innermost planet fixed, we tested $41 \times 41 \times 15 \times 5$ grid of "clones" spaced evenly across the $3 \sigma$ range in the outer planet's semi-major axis $a$, eccentricity $e$, periastron argument $\omega$, and mean anomaly $M$, respectively.

In each integration, the orbital evolution of each planet was followed until it was either ejected from the planetary system (by reaching a barycentric distance of $10 \mathrm{AU}$ ), or collided with the central body or one of the other planets. The times at which such events occurred was recorded, which allowed us to construct a map of the stability of the HD 154857 planetary system as a function of the semi-major axis and eccentricity of the outer planet. As expected, the entire $3 \sigma$ region exhibited stability for the full $10^{8} \mathrm{yr}$. Indeed, not a single ejection or collision occurred in any of the 126,075 trial systems.

\section{DISCUSSION AND CONCLUSIONS}

We have described the detection of two Jupiter-analog planets from the $15 \mathrm{yr}$ AAPS program. Our new data confirm the planetary nature of the previously unconstrained outer body in the HD 154857 system (McCarthy et al. 2004; O'Toole et al. 2007). These results highlight the importance of continuing "legacy" programs such as the AAPS, which is among the world's longest-running radial-velocity planet searches. The planets detailed in this work bring the total number of AAPSdiscovered Jupiter analogs to eight. With three Jupiter analogs confirmed in the past two years (HD 142c; Wittenmyer et al. 2012c; HD 114613b and HD 154857c, this work), the AAPS has nearly doubled its discoveries of these objects in years 14 and 15 of operation. We expect further discoveries of Jupiter analogs over the next few years as additional candidates complete orbits.

The AAPS has shifted its primary focus to the search for Jupiter analogs. Central to this strategy is the selection of a subset of $\sim 120$ targets (from the original 250 star AAPS sample) which satisfy two criteria: (1) sufficient observational baseline to detect a Jupiter analog, and (2) a sufficiently small velocity scatter to enable the robust detection of the $\sim 5-15 \mathrm{~m} \mathrm{~s}^{-1}$ signal produced by a Jupiter analog. Criterion (1) eliminates those

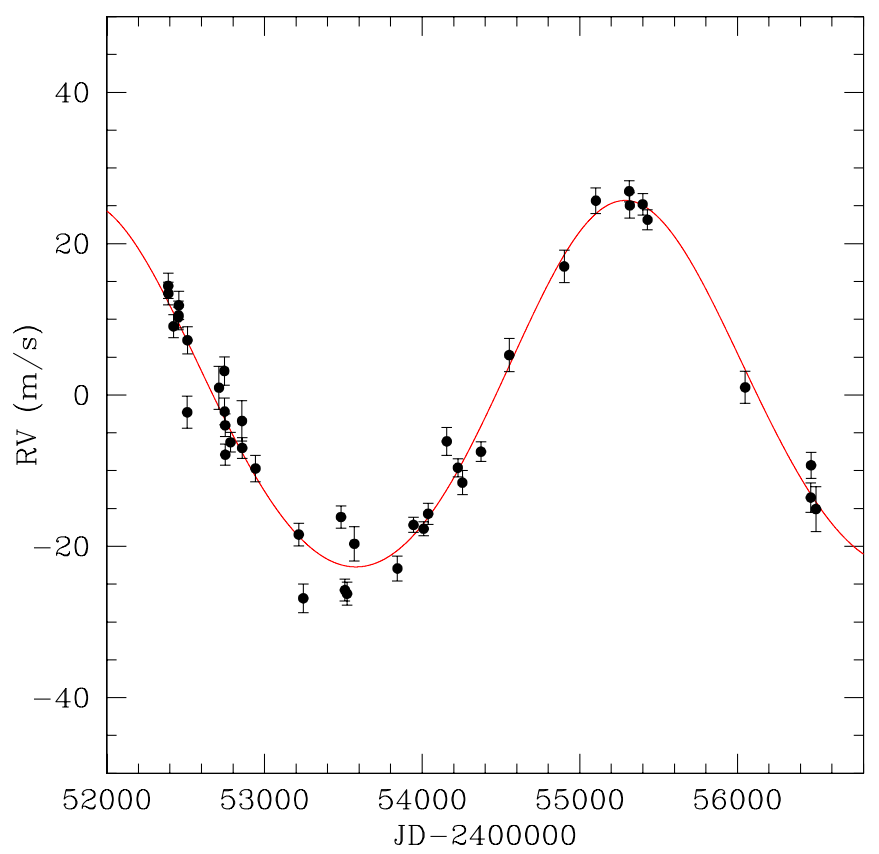

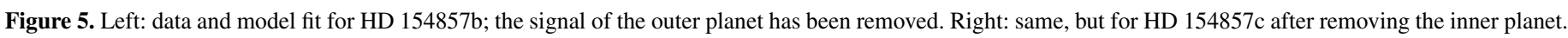
(A color version of this figure is available in the online journal.) 
stars added to the AAPS list well after its inception in 1998, and criterion (2) eliminates those stars which have high levels of intrinsic activity noise which would severely degrade the achievable detection limit. Using the detections and stringent limits from the non-detections, for every target we will be able to detect or exclude Jupiter analogs with high confidence. The result will be a direct measurement of the frequency of such objects, without suffering from significant incompleteness, which adds substantial uncertainty to this measurement (e.g., Cumming et al. 2008; Wittenmyer et al. 2011b).

There is an emerging correlation between debris disks and low-mass planets, first noted by Wyatt et al. (2012). They used Herschel to detect debris disks around four of six stars known to host only low-mass planets; no debris disks were found in the five systems hosting giant planets. One of the stars discussed here, HD 154857, has been observed for infrared excess (indicative of debris disks akin to the solar system's Edgeworth-Kuiper Belt). No excess was found from Spitzer and Herschel observations (Bryden et al. 2009; Dodson-Robinson et al. 2011). The HD 154857 system, hosting two giant planets and no detectable debris, is consistent with the pattern noted by Wyatt et al. (2012).

To obtain a complete picture of the nature of the planet candidates we have presented here, it would be ideal to determine true masses, rather than the minimum mass derived from radialvelocity measurements. Direct imaging offers a way forward: for stars known to host a long-period radial-velocity planet candidate, imaging can determine whether that object is stellar (i.e., detectable by imaging) or substellar. This type of characterization has been done for some planet candidates, such as 14 Herculis c ( $a>7$ AU; Wittenmyer et al. 2007), for which AO imaging by Rodigas et al. (2011) established an upper limit of $42 M_{\text {Jup }}$. The TRENDS survey (Crepp et al. 2012, 2013a, 2013b) is currently using this strategy to target stars with known radialvelocity trends. The Gemini Planet Imager, now installed on the $8 \mathrm{~m}$ Gemini South telescope (Hartung et al. 2013), has been specifically designed for the detection of these giant planets (McBride et al. 2011). It will provide not only the high contrasts needed to detect them, but also low-resolution spectra for each planet found, which can be used for their characterization. We are now at a convergence of two developments in exoplanetary science: (1) radial-velocity data now extend comfortably into the range of Jupiter-analog orbital periods, and (2) direct imaging techniques have improved to the point where it is possible to detect Jupiter-like planets orbiting Sun-like stars at orbital distances approaching that of our own Jupiter ( $\sim 5$ AU). These complementary techniques can bridge the detectability gap, enabling direct measurements of the occurrence rate of Jupiter analogs orbiting Sun-like stars.

We thank the referee, William Cochran, for a timely report which improved this manuscript. This research is supported by Australian Research Council grants DP0774000 and DP130102695. This research has made use of NASA's Astrophysics Data System (ADS), and the SIMBAD database, operated at CDS, Strasbourg, France. This research has also made use of the Exoplanet Orbit Database and the Exoplanet Data Explorer at www.exoplanets.org (Wright et al. 2011).

\section{REFERENCES}

Bailey, J., Butler, R. P., Tinney, C. G., et al. 2009, ApJ, 690, 743 Bond, J. C., Lauretta, D. S., Tinney, C. G., et al. 2008, ApJ, 682, 1234 Bond, J. C., Tinney, C. G., Butler, R. P., et al. 2006, MNRAS, 370, 163
Borucki, W. J., Koch, D., Basri, G., et al. 2010, Sci, 327, 977

Bryden, G., Beichman, C. A., Carpenter, J. M., et al. 2009, ApJ, 705, 1226

Butler, R. P., Marcy, G. W., Williams, E., et al. 1996, PASP, 108, 500

Butler, R. P., Wright, J. T., Marcy, G. W., et al. 2006, ApJ, 646, 505

Carter, B. D., Butler, R. P., Tinney, C. G., et al. 2003, ApJL, 593, L43

Casagrande, L., Schönrich, R., Asplund, M., et al. 2011, A\&A, 530, A138

Chambers, J. E. 1999, MNRAS, 304, 793

Crepp, J. R., Johnson, J. A., Howard, A. W., et al. 2012, ApJ, 761, 39

Crepp, J. R., Johnson, J. A., Howard, A. W., et al. 2013a, ApJ, 771, 46

Crepp, J. R., Johnson, J. A., Howard, A. W., et al. 2013b, ApJ, 774, 1

Cumming, A., Butler, R. P., Marcy, G. W., et al. 2008, PASP, 120, 531

Dawson, R. I., \& Fabrycky, D. C. 2010, ApJ, 722, 937

Diego, F., Charalambous, A., Fish, A. C., \& Walker, D. D. 1990, Proc. SPIE, 1235,562

Dodson-Robinson, S. E., Beichman, C. A., Carpenter, J. M., \& Bryden, G. 2011, AJ, 141, 11

Dumusque, X., Pepe, F., Lovis, C., et al. 2012, Natur, 491, 207

Fressin, F., Torres, G., Charbonneau, D., et al. 2013, ApJ, 766, 81

Ghezzi, L., Cunha, K., Smith, V. V., et al. 2010, ApJ, 720, 1290

Goździewski, K., Nasiroglu, I., Słowikowska, A., et al. 2012, MNRAS, 425,930

Gray, R. O., Corbally, C. J., Garrison, R. F., et al. 2006, AJ, 132, 161

Hartung, M., Macintosh, B., Poyneer, L., et al. 2013, in Proc. Third AO4ELT Conference, ed. S. Esposito \& L. Fini, 116

Hatzes, A. P. 2013, ApJ, 770, 133

Henry, T. J., Soderblom, D. R., Donahue, R. A., \& Baliunas, S. L. 1996, AJ, 111,439

Holmberg, J., Nordström, B., \& Andersen, J. 2009, A\&A, 501, 941

Horner, J., Hinse, T. C., Wittenmyer, R. A., Marshall, J. P., \& Tinney, C. G. 2012a, MNRAS, 427, 2812

Horner, J., \& Jones, B. W. 2008, IJAsB, 7, 251

Horner, J., \& Jones, B. W. 2010a, IJAsB, 9, 273

Horner, J., Jones, B. W., \& Chambers, J. 2010, IJAsB, 9, 1

Horner, J., Marshall, J. P., Wittenmyer, R. A., \& Tinney, C. G. 2011, MNRAS, 416, L11

Horner, J., Mousis, O., Petit, J.-M., \& Jones, B. W. 2009, P\&SS, 57, 1338

Horner, J., Wittenmyer, R. A., Hinse, T. C., \& Tinney, C. G. 2012b, MNRAS, 425,749

Horner, J., Wittenmyer, R. A., Hinse, T. C., et al. 2013, MNRAS, 435, 2033

Houk, N., \& Cowley, A. P. (ed.) 1975, in University of Michigan Catalogue of Two-dimensional Spectral Types for the HD Stars, Vol. 1, Declinations $-90^{\circ}$ to $-53^{\circ}$ (Ann Arbor, MI: Department of Astronomy, Univ. Michigan), 19

Howard, A. W., Marcy, G. W., Bryson, S. T., et al. 2012, ApJS, 201, 15

Jenkins, J. S., Jones, H. R. A., Tinney, C. G., et al. 2006, MNRAS, 372, 163

Jefferys, W. H., Fitzpatrick, M. J., \& McArthur, B. E. 1988, CeMec, 41, 39

Jones, H. R. A., Butler, R. P., Tinney, C. G., et al. 2010, MNRAS, 403, 1703

Kürster, M., Schmitt, J. H. M. M., Cutispoto, G., \& Dennerl, K. 1997, A\&A, 320,831

Lewis, A. R., Quinn, T., \& Kaib, N. A. 2013, AJ, 146, 16

Lomb, N. R. 1976, Ap\&SS, 39, 447

Maldonado, J., Eiroa, C., Villaver, E., Montesinos, B., \& Mora, A. 2012, A\&A, 541, A 40

McBride, J., Graham, J. R., Macintosh, B., et al. 2011, PASP, 123, 692

McCarthy, C., Butler, R. P., Tinney, C. G., et al. 2004, ApJ, 617, 575

O’Toole, S., Jones, H. R. A., Tinney, C. G., et al. 2009a, ApJ, 701, 1732

O'Toole, S., Tinney, C. G., Butler, R., et al. 2009b, ApJ, 697, 1263

O’Toole, S. J., Butler, R. P., Tinney, C. G., et al. 2007, ApJ, 660, 1636

Robertson, P., Endl, M., Cochran, W. D., et al. 2012a, ApJ, 749, 39

Robertson, P., Horner, J., Wittenmyer, R. A., et al. 2012b, ApJ, 754, 50

Rodigas, T. J., Males, J. R., Hinz, P. M., Mamajek, E. E., \& Knox, R. P. 2011, ApJ, 732, 10

Saar, S. H., \& Osten, R. A. 1997, MNRAS, 284, 803

Scargle, J. D. 1982, ApJ, 263, 835

Sousa, S. G., Santos, N. C., Mayor, M., et al. 2008, A\&A, 487, 373

Takeda, G., Ford, E. B., Sills, A., et al. 2007, ApJS, 168, 297

Tan, X., Payne, M. J., Hoi Lee, M., et al. 2013, ApJ, 777, 101

Torres, C. A. O., Quast, G. R., da Silva, L., et al. 2006, A\&A, 460, 695

Torres, G., Andersen, J., \& Giménez, A. 2010, A\&ARv, 18, 67

Valenti, J. A., Butler, R. P., \& Marcy, G. W. 1995, PASP, 107, 966

Valenti, J. A., \& Fischer, D. A. 2005, ApJS, 159, 141

van Leeuwen, F. 2007, A\&A, 474, 653

Wittenmyer, R. A., Endl, M., \& Cochran, W. D. 2007, ApJ, 654, 625

Wittenmyer, R. A., Endl, M., Cochran, W. D., et al. 2006, AJ, 132, 177

Wittenmyer, R. A., Horner, J., \& Marshall, J. P. 2013a, MNRAS, 431, 2150

Wittenmyer, R. A., Horner, J., Marshall, J. P., Butters, O. W., \& Tinney, C. G. 2012a, MNRAS, 419, 3258

Wittenmyer, R. A., Horner, J., \& Tinney, C. G. 2012b, ApJ, 761, 165 
Wittenmyer, R. A., Horner, J., Tuomi, M., et al. 2012c, ApJ, 753, 169

Wittenmyer, R. A., Tinney, C. G., Butler, R. P., et al. 2011a, ApJ, 738, 81

Wittenmyer, R. A., Tinney, C. G., Horner, J., et al. 2013b, PASP, 125, 351

Wittenmyer, R. A., Tinney, C. G., O’Toole, S. J., et al. 2011b, ApJ, 727, 102
Wright, J. T. 2005, PASP, 117, 657

Wright, J. T., Fakhouri, O., Marcy, G. W., et al. 2011, PASP, 123, 412

Wyatt, M. C., Kennedy, G., Sibthorpe, B., et al. 2012, MNRAS, 424, 1206

Zechmeister, M., \& Kürster, M. 2009, A\&A, 496, 577 